\title{
The inclusion of health data standards in the implementation of pharmacogenomics systems: a scoping review
}

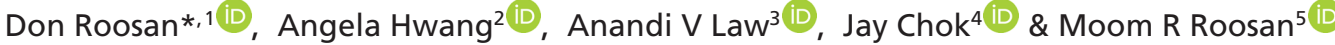 \\ ${ }^{1}$ Assistant Professor, Department of Pharmacy Practice \& Administration, College of Pharmacy, Western University of Health \\ Sciences, $309 \mathrm{E} 2^{\text {nd }}$ street, Pomona, CA 91766, USA \\ ${ }^{2}$ Research Assistant, Department of Pharmacy Practice \& Administration, College of Pharmacy, Western University of Health \\ Sciences, Pomona, CA 91766, USA \\ ${ }^{3}$ Professor, Department of Pharmacy Practice \& Administration, College of Pharmacy, Western University of Health Sciences, \\ Pomona, CA 91766, USA \\ ${ }^{4}$ Associate Professor, School of Applied Life Sciences, Keck Graduate Institute, Claremont Colleges, Pomona, CA 91711, USA \\ ${ }^{5}$ Assistant Professor, School of Pharmacy, Department of Pharmacy Practice, Chapman University, Irvine, CA 92618, USA \\ *Author for correspondence: Tel.: +1 909469 8778; droosan@westernu.edu
}

Background: Despite potential benefits, the practice of incorporating pharmacogenomics (PGx) results in clinical decisions has yet to diffuse widely. In this study, we conducted a review of recent discussions on data standards and interoperability with a focus on sharing PGx test results among health systems. Materials \& methods: We conducted a literature search for PGx clinical decision support systems between 1 January 2012 and 31 January 2020. Thirty-two out of 727 articles were included for the final review. Results: Nine of the 32 articles mentioned data standards and only four of the 32 articles provided solutions for the lack of interoperability. Discussions: Although PGx interoperability is essential for widespread implementation, a lack of focus on standardized data creates a formidable challenge for health information exchange. Conclusion: Standardization of PGx data is essential to improve health information exchange and the sharing of PGx results between disparate systems. However, PGx data standards and interoperability are often not addressed in the system-level implementation.

First draft submitted: 9 May 2020; Accepted for publication: 22 June 2020; Published online: 30 October 2020

Keywords: clinical decision support systems • health information exchanges $\bullet$ health information interoperability $\bullet$ implementation science • pharmacogenomics study

Pharmacogenomics (PGx) is well established as an important factor for improving drug efficacy and reducing adverse events [1,2]. Many studies have found PGx screenings to be cost-effective due to the decreasing cost of tests [3-5]. Moreover, a combination of economic, humanistic and clinical considerations necessitates the use of PGx as a tool to deliver optimal patient care. Identifying genetic dispositions is critical for predicting successful or failed medication therapy. With the advent of precision medicine and big data in healthcare, it is momentous to incorporate PGx for optimizing medication therapy outcomes [6-8]. Different governing bodies have established guidelines for incorporating PGx results into clinical practice due to the pivotal role PGx plays on the treatment regimen. The US FDA published biomarker and association tables to identify important drug-gene interactions easily [9]. Moreover, the NIH has funded the Pharmacogenomics Research Network (PGRN), catalyzing the formation of other resources such as PharmGKB for PGx knowledge base and PharmVar for allelic nomenclature. Several international organizations, including Clinical Pharmacogenetics Implementation Consortium (CPIC), Dutch Pharmacogenetics Working Group (DPWG) and the Canadian Pharmacogenomics Network for Drug Safety (CPNDS), aim to give clinical dosing recommendations to prescribers.

The Centers for Medicare and Medicaid Services (CMS) established the PGx payment criteria for four genes with specific drugs: CYP2C19, CYP2D6, and CYP2C9 and VKORC1 for clopidogrel, antidepressants and warfarin, respectively. The commercial payers vary in their willingness to pay for tests, and the reimbursements are based

Future Medicine 
on a case by case scenario. In 2012, current procedural terminology (CPT) codes for PGx were established for easier documentation and billing [10]. Moreover, genetic counseling is a billable service for both physicians and genetic counselors with an established CPT code [11]. As PGx testing becomes a more ubiquitous practice, reimbursement practices must follow to provide comprehensive care to patients. Therefore, the congress passed the Genetic Information Nondiscrimination Act (GINA) that prevents health insurance enrollment and employment discrimination based on genetic disposition to reassure patient protection and encourage testing for susceptible patients in 2008. State-specific GINA, such as CalGINA, further prevents discrimination in housing, education and public accommodations [12].

Health Information Technology for Economic and Clinical Health Act (HITECH) of 2009 served as an investment from the Office of the National Coordinator (ONC) to improve the use of technology within healthcare. The program incentivized providers and health systems to adopt electronic health records (EHRs) to store patient data to promote meaningful use [13]. Clinical decision support systems (CDSSs) are extensions of existing health information technology designed to aid novice and expert clinicians in medical decision-making by using evidencebased recommendations or alerts [14]. CDSSs are often integrated into EHRs for most laboratory result values ready for review at the point-of-care [15-17]. For PGx CDSSs, many studies report integrating PGx testing results into EHRs [18-20]. The Implementing Genomics in Practice (IGNITE) network successfully integrated PGx CDSSs in six different sites within the USA [18]. Each site had different ways of integrating CDSSs into the EHRs. Another functional PGx CDSS implementation is from the Mayo Clinic's RIGHT protocol. The CDSSs were integrated into the EHR for providers. Also, patients were able to review results on their online patient portals [19]. The Electronic Medical Records and Genomics (eMERGE) network supported developing algorithms for utilizing genotyping and phenotyping data electronically for PGx candidate genes in several sites across the nation. However, no universal standards exist yet for an external CDSS [21]. Many study sites, including Mayo Clinic, University of Chicago, University of Florida, eMERGE and the IGNITE Networks sites, continue to utilize and implement PGx CDSSs within the health systems. Globally, PGx CDSSs are continuously operating in various preemptive and reactive testing settings in various countries, including the European Union. Although multiple PGx CDSS implementations exist, studies describing a successfully incorporated PGx into clinical practice focus mainly on developing clinical result reporting, CDSS alerts and provider education [22,23]. Although these PGx initiatives by multiple sites have shown the feasibility of implementing PGx into clinical practice, barriers exist in the widespread PGx utilization interoperability. Given the stable nature of the germline variant data and the clinical utility of the PGx information, the highest level of interoperability is critical to facilitate a secure, seamless and timely communication of the PGx results both within and between healthcare organizations, entities and individuals.

The HITECH act encouraged the use of technology to improve patient care by integrating CDSSs into EHRs for wider adoption. Nevertheless, the interoperability becomes a concern due to the inability of each unique system to communicate with each other [24,25]. In response to this problem, the ONC added a Health Information Exchange (HIE) initiative to address interoperability between and within systems. However, such initiatives are currently still limited. The Veterans Affairs is set to launch an interoperable system for veterans who visit a non-veterans affairs care provider. The Veterans Health Information Exchange will thus allow for secure access to patient health lab results, notes and health information to community providers [26]. Other impending HIE programs are slower to progress. One of those reasons is the lack of a broader vision to incorporate data standardization [27,28].

Data standardization is particularly important in healthcare since EHR vendors represent data differently. Electronically, systems are unable to recognize the difference in data formatting, which results in the inability to exchange information. Thus, exchanging data without the use of standards risks the misinterpretation of the data. The exchange of PGx results is especially important since the results are reusable and constant throughout a patient's lifetime. If a patient wants to change health plans or primary care provider, the new healthcare provider should have access to previously tested PGx results. The lack of data standardization prevents results from being shared electronically between different health systems. To facilitate the HIE process, CPIC term standardization efforts have lead to the development of LOINC and SNOMED terms. Even with the data standards in genomics, implementation of PGx-specific standards are not prevalent. Many studies expressed the need for fully developed PGx data standardization [29-31]. Therefore, data standards in laboratory processes, reporting of the results, and reimbursement processes are crucial for achieving PGx data interoperability.

Clinical workflow recommendations have been incorporated into CDSS prototypes due to the emerging relevance and importance of PGx tests. PGx CDSSs provide a knowledge source and education for clinicians, create clinical recommendations and ensure dosing adjustments based on PGx test results and alert notifications [22,32-34]. 
However, a gap exists in the PGx CDSS literature. Many papers and reviews discuss PGx integration solely into EHRs without CDSSs or discuss CDSSs without considering data standards. Articles mention the need for data standardization in PGx; however, do not go in-depth about possible solutions for the future of health information exchange [35]. Our study aims to review PGx CDSSs piloted or prototyped and seeks to determine if interoperability between different EHRs was discussed or addressed. The Preferred Reporting Items for Systematic Reviews and Meta-Analysis (PRISMA) guidelines were utilized to report this review [36].

\section{Materials \& methods}

The literature search focused on articles describing PGx implementation with CDSSs. The inclusion criteria for the search for articles had two main focus: articles related to PGx system implementation and articles related to PGxrelated protocol or module implementations in a decision support system or EHRs. Exclusion criteria were PGx implementation into health systems or utilizing PGx reports for clinical decisions without a CDSS, review articles, abstracts, conference posters, brief reports, case reports, correction communications, rapid communications, posters or abstracts, articles not in English and research describing neither PGx nor CDSS. The risk of bias was not assessed; however, similar study-locations were grouped together.

\section{Literature search}

A literature search was conducted in January 2020 for articles published between 1 January 2012 and 31 January 2020 using five databases: PubMed, Web of Science, Google Scholar, Scopus and Embase. The keywords used in the PubMed search query included ('clinical decision support' [MeSH Terms] or 'decision support' or 'decision making') and ('pharmacogenetics' [MeSH Terms] or 'pharmacogenetic' or 'pharmacogenomic'). A PGx CDSS for even a single gene was included and screened for eligibility. Two reviewers independently screened titles, keywords and abstracts of articles for relevance and duplications. Both reviewers analyzed full-text articles and discussed the disagreements for consensus. Figure 1 summarizes the article selection process.

\section{Data collection}

Data standards for CDSSs were assessed in the full-text articles that met the inclusion criteria. Data items abstracted included 'system or project name', 'study location', 'data standard for PGx CDSS', 'interoperability summary' and 'system-related interoperability features in the PGx CDSS. The PGx system name was identified throughout the article as the project name. If there was none mentioned, it was listed as 'no name'. The study location was the main geographic location of where the CDSS study was conducted [23]. Data standards for PGx CDSS were defined as systems or rules adhered to standardize reporting of PGx data, which includes Health Level Seven (HL7) as it relates to interoperability. The standardization of laboratory reports, variants reporting or clinical recommendations was not included in the definition for the purposes of this review. The interoperability summary was abstracted from direct quotes addressing interoperability in each article. Finally, interoperability features and designs were summarized to understand the extent of the health information exchange implemented into the CDSSs. The findings were summarized in two tables with descriptive narratives.

\section{Results}

A cumulative of 727 articles were identified from all five databases. Of these, 650 articles were excluded by title and abstract screening for duplication or describing a CDSS not pertaining to PGx or PGx-related medication data. Seventy-seven full-text articles were reviewed. Fort-five articles were excluded due to three reasons: 17 articles were not full-text articles and were conference abstracts, 19 studies did not describe a PGx CDSS and the remaining nine articles did not integrate actual PGx data into the CDSS. Finally, 32 articles were evaluated in this review.

\section{Data standards}

Nine of the 32 studies mentioned data standards, such as HL7, and all but two utilized HL7 to report results into EHR or external systems, as detailed in Table 1 [29,31,37-43]. The remaining 23 articles mentioned standardized guidelines in regard to clinical recommendations, like CPIC and DPWG, but were unrelated to standardizing reporting of results [19,20,30,34,44-61]. In particular, Bousman et al. described four different PGx CDSSs from different companies to determine the consistency and accuracy of results. Overall, $58 \%$ of medication recommendations from the four PGx CDSSs reported similar results. Forty-two percent differed in their representation or clinical recommendations of results. Additionally, among patients with actionable genes identified by CDSS flags, $19 \%$ of 
Table 1. Results of thirty-two articles describing pharmacogenomics clinical decision support systems, data standards and interoperability.

\begin{tabular}{|c|c|c|c|}
\hline System or project name & Study location & Data standards for PGx CDSS & Ref. \\
\hline 1. TPMT CDS testing & Mayo Clinic, Rochester, MN, USA & HL7 & [43] \\
\hline 2. PGTIC & $\begin{array}{l}\text { National Institutes of Health Clinical } \\
\text { Center, Bethesda, MD, USA }\end{array}$ & HL7, Arden Syntax & [39] \\
\hline 3. PGTIC & $\begin{array}{l}\text { National Institutes of Health Clinical } \\
\text { Center, Bethesda, MD, USA }\end{array}$ & HL7, Arden Syntax & [40] \\
\hline 4. GACS & Elimu Informatics, Richmond, CA, USA & HL7 FHIR & [41] \\
\hline 5. SHARPC-2B & $\begin{array}{l}\text { Arizona State University, Scottsdale, AZ, } \\
\text { USA }\end{array}$ & HL7, UML & [29] \\
\hline 6. FARMAPRICE & Aviano Hospital, Aviano Italy & HL7, IHE, DICOM, XDS & [42] \\
\hline 7. The U-PGx project & $\begin{array}{l}\text { Various institutions across } \\
\text { seven different countries: Austria, } \\
\text { Spain, Greece, Great Britain, Italy, } \\
\text { Slovenia and The Netherlands }\end{array}$ & HL7 FHIR, HTTPS & [37] \\
\hline 8. PREDICT (2012) & $\begin{array}{l}\text { Vanderbilt University School of } \\
\text { Medicine, Nashville, TN, USA }\end{array}$ & $\begin{array}{l}\text { HL7 } \\
\text { Peterson et al. mentioned that they will } \\
\text { integrate HL7 in the future when } \\
\text { standardization is better developed; } \\
\text { Pulley et al. did not mention standards }\end{array}$ & [48] \\
\hline 9. PREDICT (2013) & $\begin{array}{l}\text { Vanderbilt University School of } \\
\text { Medicine, Nashville, TN, USA }\end{array}$ & $\begin{array}{l}\text { Peterson et al. mentioned that they will } \\
\text { integrate HL7 in the future when } \\
\text { standardization is better developed; } \\
\text { Pulley et al. did not mention standards }\end{array}$ & [31] \\
\hline 10. GPS portal (2012) & University of Chicago, Chicago, IL, USA & $\begin{array}{l}\text { Danahey et al. mentioned HL7 SMART } \\
\text { on FHIR but did not integrate into } \\
\text { system; all others did not mention } \\
\text { standards }\end{array}$ & [53] \\
\hline 11. GPS (2014) & University of Chicago, Chicago, IL, USA & $\begin{array}{l}\text { Danahey et al. mentioned HL7 SMART } \\
\text { on FHIR but did not integrate into } \\
\text { system; all others did not mention } \\
\text { standards }\end{array}$ & [59] \\
\hline 12. GPS (2014) & University of Chicago, Chicago, IL, USA & $\begin{array}{l}\text { Danahey et al. mentioned HL7 SMART } \\
\text { on FHIR but did not integrate into } \\
\text { system; all others did not mention } \\
\text { standards }\end{array}$ & [59] \\
\hline 13. GPS (2017) & University of Chicago, Chicago, IL, USA & $\begin{array}{l}\text { Danahey et al. mentioned HL7 SMART } \\
\text { on FHIR but did not integrate into } \\
\text { system; all others did not mention } \\
\text { standards }\end{array}$ & {$[34,38]$} \\
\hline 14. No name & $\begin{array}{l}\text { Emory University School of Medicine, } \\
\text { Atlanta, GA, USA }\end{array}$ & $\begin{array}{l}\text { Discussed the need for data } \\
\text { standardization but did not mention } \\
\text { standards }\end{array}$ & [30] \\
\hline 15. CPS & $\begin{array}{l}\text { Boston Children's Hospital (BCH), } \\
\text { Boston, MA, USA }\end{array}$ & None & [63] \\
\hline 16. MSC (2014) & $\begin{array}{l}\text { University of Vienna, Vienna, Austria } \\
\text { University of Pittsburgh, Pittsburgh, PA, } \\
\text { USA }\end{array}$ & None & [44] \\
\hline 17. MSC (2015) & $\begin{array}{l}\text { University of Pittsburgh, Pittsburgh, PA, } \\
\text { USA }\end{array}$ & & [45] \\
\hline 18. UW-PowerChart ${ }^{\circledR}$ prototype & $\begin{array}{l}\text { University of Washington, Seattle, WA, } \\
\text { USA }\end{array}$ & None & [46] \\
\hline 19. RIGHT & Mayo Clinic, Rochester, MN, USA & None & [19] \\
\hline 20. Right Drug, Right, Dose, Right Time & Mayo Clinic, Rochester, MN, USA & None & [47] \\
\hline 21. PMP & $\begin{array}{l}\text { Cleveland Clinic Health System, } \\
\text { Cleveland, OH, USA }\end{array}$ & None & [62] \\
\hline 22. PMP & $\begin{array}{l}\text { University of Florida, Gainesville, FL, } \\
\text { USA }\end{array}$ & None & [50] \\
\hline
\end{tabular}

CDSS: Clinical decision support system; CPS: Clinical pharmacogenomic service; DICOM: Digital Imaging and Communications in Medicine; FHIR: Fast healthcare interoperability resources; GACS: Genomic Archiving and Communication System; GPS: Genomic prescribing system; HL7: Health level seven; HTTPS: Hypertext transfer protocol secure; IHE: Integrating the healthcare enterprise; MSC: Medicine Safety Code; PGTIC: Pharmacogenetics Testing Implementation Committee; PGx: Pharmacogenomic; PMP: Personalized Medication Program; PREDICT: Pharmacogenomic Resource for Enhanced Decisions in Care and Treatment; SMART: Substitutable Medical Applications and Reusable Technologies; UML: Unified Modeling Language; U-PGX: Ubiquitous Pharmacogenomic; XDS: Cross-enterprise document sharing. 
Table 1. Results of thirty-two articles describing pharmacogenomics clinical decision support systems, data standards and interoperability (cont.).

\begin{tabular}{|c|c|c|c|}
\hline System or project name & Study location & Data standards for PGx CDSS & Ref. \\
\hline 23. PG4KDS (2014) & $\begin{array}{l}\text { St. Jude Children Research Hospital, } \\
\text { Memphis, TN, USA }\end{array}$ & None & [51] \\
\hline 24. PG4KDS (2014) & $\begin{array}{l}\text { St. Jude Children Research Hospital, } \\
\text { Memphis, TN, USA }\end{array}$ & None & [52] \\
\hline 25. CLIPMERGE PGx & $\begin{array}{l}\text { Icahn School of Medicine at Mount } \\
\text { Sinai, New York, USA }\end{array}$ & None & {$[21]$} \\
\hline 26. TreatGx & $\begin{array}{l}\text { University of British Columbia, } \\
\text { Vancouver, BC, Canada }\end{array}$ & None & [54] \\
\hline 27. CDST Youscript (2017) & $\begin{array}{l}\text { White County Medical Center, Searcy, } \\
\text { AR, USA }\end{array}$ & None & [55] \\
\hline 28. CDST Youscript (2018) & Magellan Health, Salt Lake City, UT, USA & None & {$[56]$} \\
\hline 29. PGx CDS & $\begin{array}{l}\text { University of Washington, Seattle, WA, } \\
\text { USA }\end{array}$ & None & [57] \\
\hline 30. eMERGE-PGx project & Multiple sites within the USA & None & [20] \\
\hline 31. CYP-GUIDES & Hartford Hospital, Hartford, CT, USA & None & [58] \\
\hline $\begin{array}{l}\text { 32. The Implementation of } \\
\text { Pharmacogenetics into Primary care } \\
\text { Project (IP3 study) }\end{array}$ & $\begin{array}{l}\text { Leiden University Medical Center, The } \\
\text { vicinity of Leiden, The Netherlands }\end{array}$ & None & [60] \\
\hline \multicolumn{4}{|c|}{$\begin{array}{l}\text { CDSS: Clinical decision support system; CPS: Clinical pharmacogenomic service; DICOM: Digital Imaging and Communications in Medicine; FHIR: Fast healthcare interoperability resources; } \\
\text { GACS: Genomic Archiving and Communication System; GPS: Genomic prescribing system; HL7: Health level seven; HTTPS: Hypertext transfer protocol secure; IHE: Integrating the health- } \\
\text { care enterprise; MSC: Medicine Safety Code; PGTIC: Pharmacogenetics Testing Implementation Committee; PGx: Pharmacogenomic; PMP: Personalized Medication Program; PREDICT: } \\
\text { Pharmacogenomic Resource for Enhanced Decisions in Care and Treatment; SMART: Substitutable Medical Applications and Reusable Technologies; UML: Unified Modeling Language; } \\
\text { U-PGx: Ubiquitous Pharmacogenomic; XDS: Cross-enterprise document sharing. }\end{array}$} \\
\hline
\end{tabular}

\section{Table 2. Interoperability features and designs used in pharmacogenomics clinical decision support systems.}

System or project Interoperability summary System-related interoperability features in the PGX CDSS

The study aimed to address interoperability by ensuring sharing

- Secure data transfer of PGx results and dosing recommendations $P G x$ results within and between different healthcare settings in (HL7, .CSV, HTTPS, FHIR and RESTful) into EHR different European countries. They recognized improving

- PGx report in PDF or ODF into patient's paper based or digital file preemptive PGx testing.

- Medication Safety-Code card for facilities without EHR

GACS The authors stated that genetic sequencing data and EHR data standards have independently advanced creating a bigger issue of interoperability. In order to address this gap GACS was utilized to store data and the authors proposed using CDS Hooks, PGx CDSS that can be rendered.

SHARPC-2B

External data storage in GACS and results expressed in FHIR and CDS hooks

CDS hooks notify the external GACS to receive or generate PGx data and communicate back to EHR.

The authors published a guideline for PGx in order to combine PGx guideline using:

- UML diagrams standards to create a structured document that can be shared with the machine-readability. The guideline aims to be a starting point for PGx CDS for evidence-based care delivery.

- UML diagrams
- HL7 VMR, LS DAM and HL7 RIM
- RxNorm, NDF-RT, SNOMED CT and LOINC

FARMAPRICE The study recognized that even in a country with universal healthcare, the healthcare technology infrastructure may be still fragmented as evidenced by different EHRs throughout the Italy. The lack of a single EHR was described as a barrier to interoperability and sharing healthcare data.

\section{Open source solutions include:}

- Protected Health Information technology

- Independent from the underlying operating system

- Reports results on the latest open standards (HL7, IHE, DICOM and XDS)

CDS: Clinical decision support; CDSS: Clinical decision support systems; csv: Comma-separated values; DICOM: Digital imaging and communications in medicine; EHR: Electronic Health Record; FHIR: Fast healthcare interoperability resources; GACS: Genomic archiving and communication system; GIMS: Genetic information management suite; HL7: Health level seven; HTTPS: Hypertext transfer protocol secure; IHE: Integrating the healthcare enterprise; LOINC: Logical observation identifiers names and codes; LS DAM: Life sciences domain analysis model; NDF-RT: National drug file - reference terminology; ODF: Open document format; PDF: Portable document format; RIM: Reference information model; RESTful: Representation State Transfer; SNOMED CT: Systemized Nomenclature of Medicine - clinical terms; UML: Unified modeling language; vMR: Virtual medical record; XDS: Cross-enterprise document sharing.

those flags reported different clinical drug dosing recommendations for the same PGx variant [30]. Although many studies did not discretely state the reporting PGx data standards used, most studies recognized the lack of data standardization leading to the creation of individual protocols and standards [19,20,31,47,49-51,62]. 


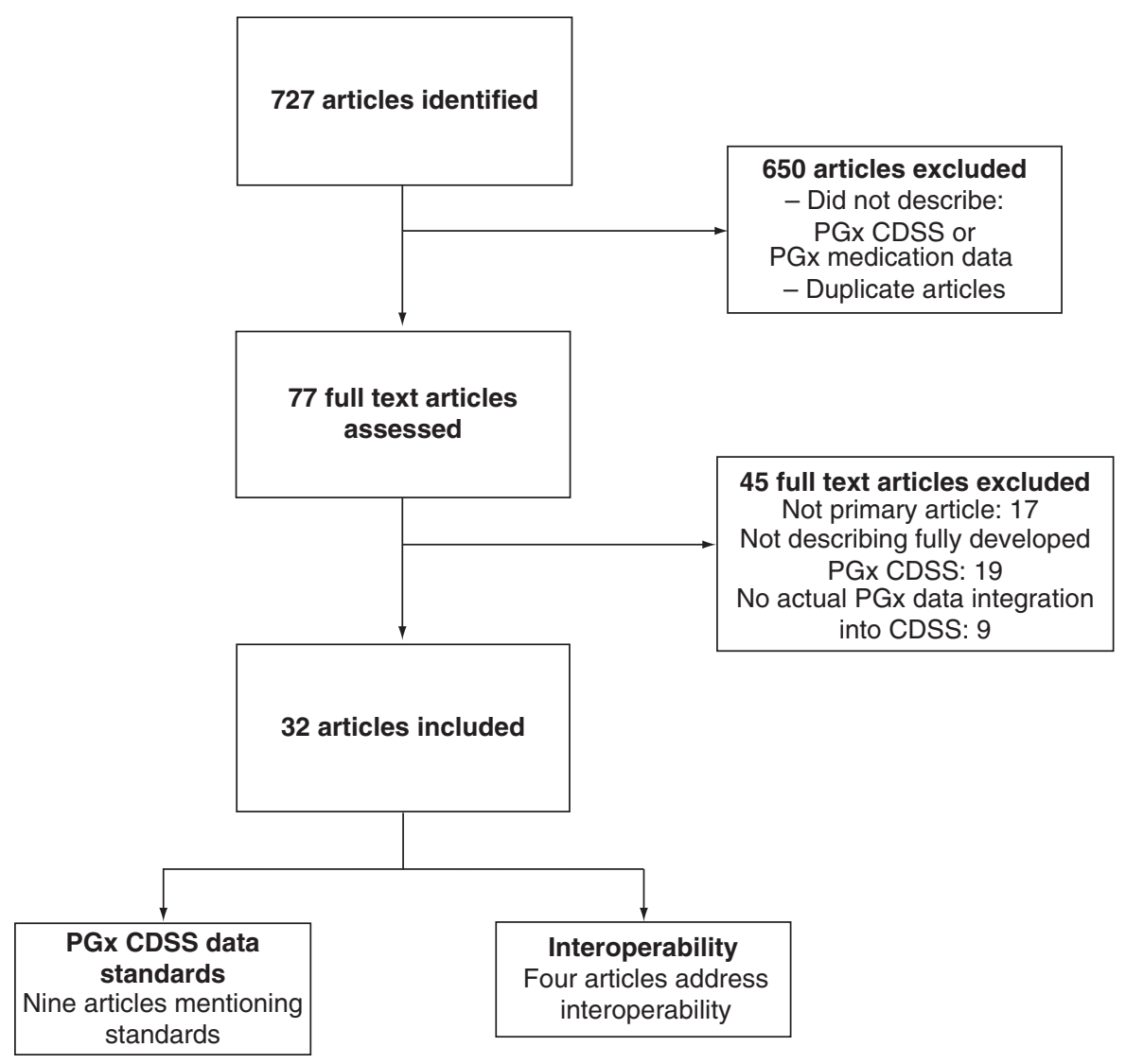

Figure 1. Literature search and process of article selection. CDSS: Clinical decision support system; PGx: Pharmacogenomics.

\section{Interoperability}

Out of the 32 articles evaluated, eight articles mentioned interoperability to a varying extent. Four briefly mentioned the need for data standardization and interoperability. These four studies did not discuss data standards in detail but considered it as a challenge while creating PGx CDSSs. The remaining four studies addressed interoperability by creating prototypes and implementing features into the system as solutions. The details of data standards used in response to the lack of data standardization in these studies are shown in Table 2.

Dolin $e t$ al. and Blagec et al. presented a similar approach to health information exchange by using an external genomic system as a source of CDSS [37,41]. The U-PGx project was created to implement PGx CDSSs in seven different European countries by using a web-based knowledge source called Genetic Information Management Suite (GIMS) [37]. GIMS translates genomic data into clinically actionable recommendations, stores results and serves as a knowledge base. For result reporting, GIMS creates reports compatible with different reporting standards (HL7, Fast Healthcare Interoperability Resources [FHIR] and Hypertext Transfer Protocol Secure [HTTPS]) and sends results to EHRs. For sites that lack an EHR, a medication safety code was created in order to review the PGx results on a web site. Furthermore, U-PGx aimed to keep records into patient's medical files using a portable document for paper-based reports or open document format for electronic files. The combination of using GIMS and medication safety code enabled the sharing of results not only within and between systems but also across countries. As of June 2017, GIMS was fully functional and being continuously utilized in three different countries.

Similar to the U-PGx project, Genomic Archiving and Communication System (GACS) serves as an external functional prototype for PGx CDSS [41]. GACS houses complete DNA sequencing data and can retrieve genetic information and feed into the EHR through clinical decision support (CDS) Hooks. CDS Hooks are application programming interfaces that use the 'hook' method for invoking CDS rules to enable communication between an external decision system and the EHR. A PGx CDSS was modeled alongside GACS to provide clinical recommen- 
dations based on the genetic information from GACS by using FHIR. In January 2018, this demonstration was completed using GACS and the PGx CDSS for TPMT and azathioprine.

The FARMAPRICE study created a prototype that used an open-source Protected Health Information Technology that operates independently as a web-based platform and has the potential to be integrated into EHRs [42]. FARMAPRICE CDSS was created in response to the lack of a standardized EHR platform to share results between health systems across Italy. Thus, the application supports reporting results in the latest standards (HL7, Integrating the Healthcare Enterprise [IHE] and Digital Imaging and Communications in Medicine [DICOM]), ensuring the interchange of PGx results between different operating systems for future use. The prototype is currently under further research and developmental use at Aviano Hospital in Italy.

In the SHARPc-2B project, the authors created a PGx guideline to combine standards (Unified Modeling Language [UML], HL7 vMR and terminology) and a shareable PGx CDSS using the ONC supported Health eDecisions (HeD) interchange format [29]. The PGx CDSS guideline by $\mathrm{HeD}$ was created using UML, SNOMED CT, RxNorm and LOINC. Using HeD, the authors were able to create a model for $H L A-B$ and abacavir that could be utilized in health systems desiring to integrate PGx into clinical practice with a standardized guideline.

\section{Discussion}

PGx research has broadened over time to include studying PGx tests as a clinical component of patient care within the context of CDSS integrated with EHRs. The studies that have been published within the last 8 years have mainly focused on CDSS alerts, developments or features $[23,33]$. Although many CDSS have been created to offer CDS to providers in patients with actionable genotypes, the discussion of data standards and interoperability has been limited [64]. Some of the data standards mentioned within the articles included SNOMED CT, LOINC, HL7, DICOM and IHE. However, most of the discussions were only limited to mentioning the use of standardized terminology. On the contrary, genomics data have been standardized based on the need to compare thousands of different datasets [65]. As a result, genomics data have been standardized to be shared, managed and analyzed. An example of genomic data standards includes the HL7 V3 Clinical Genomics Standard Specifications, which combines raw genomic data to a patient phenotype [66]. Additionally, the Genomic Knowledge Standards Work Stream (GKSWS) group works to standardize genomic data to improve compatibility with healthcare standards (HL7 and FHIR). Similarly, PGx data must be standardized as genomics data for smooth information exchange between health systems. Yet, PGx standardization has been slow to progress through continuous development. Only CPIC discussed and published standardized data to identify PGx terms for allele functions and associated phenotypes. Moreover, the improvement in standardization was furthered as SNOMED CT incorporated the standardized terms created by CPIC [67,68]. The final study we reviewed was a guideline to model PGx CDSS into an $\mathrm{ONC}$ initiated $\mathrm{HeD}$ schema. In creating the guideline, standard terminologies were used such as RxNorm, LOINC and SNOMED CT. However, the authors noted that codes for adverse drug reactions of abacavir and genotype test results were unavailable. As a result, the authors concluded that harmonizing standards for PGx was underdeveloped.

Data standardization is necessary for creating interoperable systems for PGx. Over the last 11 years, the government has been investing in converting healthcare into a more efficient and cost-saving system with the use of technology. Investments from HITECH and HIE from the US government has funneled more than 2 billion dollars into the health care system [13]. Without widespread data standardization adoption, this investment would be unproductive. Our literature search has shown that among the papers addressing PGx integration into clinical care through CDSSs, only four had taken interoperability into account. Although each study addressed interoperability with different aspects and system features, none of these studies reported data exchange mechanism between different systems. Only the U-Pgx project addressed various data exchange scenarios, including non-electronic data sharing. However, the U-Pgx project did not adequately address utilizing a standardized dataset for PGx.

Data standardization approval requires lengthy reviews and testing periods. Through an accredited collaborative process involving various stakeholders and users, Standards Development Organizations (SDOs) define, maintain and update standards. Prominent SDOs in the US healthcare sector are HL7, FHIR, Accredited Standards Committee X12 (ASC X12), Integrating the Healthcare Enterprise (IHE) and International Organization for Standardization (ISO). Usually, based on business needs the SDO decides to develop a standard. This development process enters rounds of voting, feedback, piloting the standard and making appropriate changes taking anywhere from 2 to 3 years [69]. Studies indicated the importance of standardized terms required for electronic transfer, which has been insufficiently expressed through contemporary methods [70]. Specifically for PGx data standardization, 
CPIC standardized terms have been supported as LOINC identifiers, SNOMED codes and tested by the CAP proficiency testing programs. Standardization in the genetic testing platform, alleles/variants included in the testing, reporting of the PGxs results, genotype to phenotype translation is still under development [71]. Moreover, FHIR strategies such as Substitutable Medical Applications and Reusable Technologies (SMART) would be adaptive technologies to support the flexibility of our knowledge evolution and informatics needs in the PGx realm. Therefore, the next phase should be to standardize the format of the document architecture and secure transmission of the data [72]. Also, users and SDOs should continue developing PGx data standards by collaborating with expert opinion leaders.

Standardizing PGx test results data plays a significant role in interoperability. Additionally, data standards allow the uniformity of data that can be both machine- and human-readable and used worldwide. For example, healthcare officials are using standardized data to monitor current COVID-19 pandemic cases effectively to inform the public about the gravity of the situation. Thus, data standards affect not only healthcare providers but also average people. Few data standards to consider for public health information exchange include Clinical Document Architecture (CDA) for the structure of clinical documents, HL7 for exchanging messages, FHIR for data transfer security. In the future, if PGx data can be standardized, sharing the genomic and the medication data have the potential to improve healthcare even further.

PGx results are clinically actionable throughout a patient's lifetime. With PGx data standardization, patients will be able to utilize their results anywhere they are treated. As mentioned in multiple studies, usability is an important aspect of a stand-alone patient managed genomics system. Therefore, graphical user interfaces based on user-centered design have the potential to engage both the providers and the patients [73,74]. An intuitive interface for managing PGx test results can reduce the cognitive burden on providers and patients as well as simplify the complexity in managing health information [75]. A patient managed genomics system may have usability features such as simple navigation, cognitive design, viewing controls, secure sharing options, and embedded analytics for empowering patients $[38,42,76,77]$.

Creating a PGx CDSS, which is shareable within and between different operating EHRs and other systems, will increase PGx data accessibility and utilization. As a result, PGx and medication information can be shared with different healthcare providers. For example, pharmacists in the community pharmacies may use this information to identify drug-gene interactions and recommend alternative therapies in collaboration with physicians. Moreover, clinicians can use patients PGx information to optimize medication therapy, reduce adverse drug events and improve medication adherence.

\section{Future perspective}

To summarize, our literature search suggests that PGx interoperability is a complex phenomenon mired by numerous challenges related to a well-recognized need for PGx data standardization. The crucial gap missing in the literature was the need for specific and workable features of proposed interoperable PGx CDSSs. In particular, current discussions about interoperability within PGx CDSS are insufficient. Most studies discuss the need for data standardizations but do not discuss which standards were used or referenced. We recommend that future PGx CDSS implementation research discuss the importance of data standards, including standardized PGx terminology, health information exchange and interoperability.

Acknowledgments

The authors are grateful to the pharmacy professional students and J Moon for helping with data collection.

Financial \& competing interests disclosure

The study was funded from an internal grant from Western University of Health Sciences. The authors have no other relevant affiliations or financial involvement with any organization or entity with a financial interest in or financial conflict with the subject matter or materials discussed in the manuscript apart from those disclosed.

No writing assistance was utilized in the production of this manuscript. 


\section{Executive summary}

- The lack of pharmacogenomics (PGx) data standards is a barrier to interoperability and widespread sustainable utilization of PGx data.

- Healthcare data standards and interoperability in PGx clinical decision support systems (CDSS) have not been properly addressed in the literature.

- A scoping literature review was done for articles related to PGx system implementation with a decision support system in electronic health records to explore the depth of discussion on data standards in PGx.

- Of the 32 articles that fit the inclusion criteria, only four discussed details about the lack of interoperability and system designs to overcome the lack of data standards.

- Current discussions about PGx data standardization are insufficient in the literature.

- Without further development into PGx data standards, patients and providers will not be able to utilize PGx results efficiently.

- A patient managed pharmacogenomics system sharing PGx data with clinicians and family members may ultimately empower patients to manage their own medication information.

- As more research into data standards progress, a shareable PGx platform could increase PGx utilization to promote precision medicine.

\section{References}

1. Relling MV, Evans WE. Pharmacogenomics in the clinic. Nature 526(7573), 343-350 (2015).

2. Evans WE, Relling MV. Moving towards individualized medicine with pharmacogenomics. Nature 429(6990), 464-468 (2004).

3. Berm EJ, Looff M, Wilffert B et al. Economic evaluations of pharmacogenetic and pharmacogenomic screening tests: a systematic review. second update of the literature. PLoS ONE 11(1), e0146262 (2016).

4. Winner JG, Carhart JM, Altar CA et al. Combinatorial pharmacogenomic guidance for psychiatric medications reduces overall pharmacy costs in a 1 year prospective evaluation. Curr. Med. Res. Opin. 31(9), 1633-1643 (2015).

5. Gold HT, Hall MJ, Blinder V, Schackman BR. Cost effectiveness of pharmacogenetic testing for uridine diphosphate glucuronosyltransferase 1A1 before irinotecan administration for metastatic colorectal cancer. Cancer 115(17), 3858-3867 (2009).

6. Roosan D, Karim M, Chok J, Roosan M. Operationalizing healthcare big data in the electronic health records using a heatmap visualization technique. Proceedings of the 13th International Joint Conference on Biomedical Engineering Systems and Technologies - Volume 5 HEALTHINF: HEALTHINF 361-368 (2020).

7. Wang Y, Kung L, Wang W, Cegielski C. An integrated big data analytics-enabled transformation model: application to health care. Inform. Manage. 55(1), 64-79 (2018).

8. Beam A, Is K. Big data and machine learning in health care. JAMA 319(13), 1317-1318 (2018).

9. Health $\mathrm{C}$ for $\mathrm{D}$ and $\mathrm{R}$. Table of pharmacogenetic associations. (2020). http://www.fda.gov/medical-devices/precision-medicine/table-pharmacogenetic-associations

10. Hefti E, Blanco J. Documenting pharmacogenomic testing with current procedure terminology (CPT) codes, a review of past and present practices. J. AHIMA 87(1), 56-59 (2016).

11. Gustafson SL, Pfeiffer G, Eng C. A large health system's approach to utilization of the genetic counselor CPT(R) 96040 code. Genet. Med. 13(12), 1011-1014 (2011).

12. Singh P, Mcintyre A, O'Connor S. Increasing use of genetic data requires new privacy considerations. J. Sci. Policy Governance 15(1), 6 (2019).

13. Blumenthal D. Launching HITECH. N. Engl. J. Med. 361(5), 382-385 (2010).

14. Islam R, Mayer J, Clutter J. Supporting novice clinicians cognitive strategies: system design perspective. IEEE EMBS Int. Conf. Biomed. Health Inform. 2016, 509-512 (2016).

15. Islam R, Weir C, Del Fiol G. Heuristics in managing complex clinical decision tasks in experts' decision making. IEEE Int. Conf. Healthc. Inform. 2014, 186-193 (2014).

16. Islam R, Weir CR, Jones M, Del Fiol G, Samore MH. Understanding complex clinical reasoning in infectious diseases for improving clinical decision support design. BMC Med. Inform. Decis. Mak. 15(1), 101 (2015).

17. Roosan D, Samore M, Jones M, Livnat Y, Clutter J. Big-data based decision-support systems to improve clinicians' cognition. IEEE Int. Conf. Healthc. Inform. 2016, 285-288 (2016).

18. Weitzel KW, Alexander M, Bernhardt BA et al. The IGNITE network: a model for genomic medicine implementation and research. BMC Med. Genomics 9, 1 (2016).

19. Bielinski SJ, Olson JE, Pathak J et al. Preemptive genotyping for personalized medicine: design of the right drug, right dose, right time-using genomic data to individualize treatment protocol. Mayo Clin. Proc. 89(1), 25-33 (2014). 
20. Rasmussen-Torvik LJ, Stallings SC, Gordon AS et al. Design and anticipated outcomes of the eMERGE-PGx project: a multicenter pilot for preemptive pharmacogenomics in electronic health record systems. Clin. Pharmacol. Ther. 96(4), 482-489 (2014).

21. Gottesman O, Kuivaniemi H, Tromp G et al. The Electronic Medical Records and Genomics (eMERGE) network: past, present, and future. Genet. Med. 15(10), 761-771 (2013).

22. Welch BM, Kawamoto K. Clinical decision support for genetically guided personalized medicine: a systematic review. J. Am. Med. Inform. Assoc. 20(2), 388-400 (2013).

23. Hinderer M, Boeker M, Wagner SA et al. Integrating clinical decision support systems for pharmacogenomic testing into clinical routine - a scoping review of designs of user-system interactions in recent system development. BMC Med. Inform. Decis. Mak. 17(1), 81 (2017).

24. Hsiao C, Hing E. Use and characteristics of electronic health record systems among office-based physician practices: United States, 2001-2013. NCHS Data Brief(143), 8 (2014).

25. Henry J, Pylypchuk Y, Searcy T, Patel V. Office of the National Coordinator for Health Information Technology. Adoption of electronic health record systems among U.S. Non-Federal Acute Care Hospitals: 2008-2015. ONC Data Brief. Washington DC, USA (2016). https://dashboard.healthit.gov/evaluations/data-briefs/non-federal-acute-care-hospital-ehr-adoption-2008-2015.php

26. Jason C. EHR intelligence. VA preparing for april launch of health information exchange. https://ehrintelligence.com/news/va-preparing-for-april-launch-of-health-information-exchange

27. Richesson RL, Krischer J. Data standards in clinical research: gaps, overlaps, challenges and future directions. J. Am. Med. Inform. Assoc. 14(6), 687-696 (2007).

28. Wu H, Larue E. Barriers and facilitators of health information exchange (HIE) adoption in the United States. 2015 48th Hawaii International Conference on System Sciences. 2942-2949 (2015).

29. Linan M, Sottara D, Freimuth RR. Creating shareable clinical decision support rules for a pharmacogenomics clinical guideline using structured knowledge representation. AMIA 2015, 1985-1994 (2015).

30. Bousman CA, Dunlop BW. Genotype, phenotype, and medication recommendation agreement among commercial pharmacogenetic-based decision support tools. Pharmacogenomics J. 18(5), 613-622 (2018).

31. Peterson JF, Bowton E, Field JR et al. Electronic health record design and implementation for pharmacogenomics: a local perspective. Genet. Med. 15(10), 833-841 (2013).

32. Nutescu EA, Drozda K, Bress AP et al. Feasibility of implementing a comprehensive warfarin pharmacogenetics service. Pharmacotherapy 33(11), 1156-1164 (2013).

33. Empey PE, Stevenson JM, Tuteja $S$ et al. Multisite investigation of strategies for the implementation of CYP2C19 genotype-guided antiplatelet therapy. Clin. Pharmacol. Ther. 104(4), 664-674 (2018).

34. O'Donnell PH, Wadhwa N, Danahey K et al. Pharmacogenomics-based point-of-care clinical decision support significantly alters drug prescribing. Clin. Pharmacol. Ther. 102(5), 859-869 (2017).

35. Dunnenberger HM, Crews KR, Hoffman JM et al. Preemptive clinical pharmacogenetics implementation: current programs in five US medical centers. Annu. Rev. Pharmacol. Toxicol. 55, 89-106 (2015).

36. Moher D, Liberati A, Tetzlaff J, Altman DG. Group P. Preferred reporting items for systematic reviews and meta-analyses: the PRISMA statement. PLoS Med. 6(7), e1000097 (2009).

37. Blagec K, Koopmann R, Crommentuijn-van Rhenen M et al. Implementing pharmacogenomics decision support across seven European countries: the ubiquitous pharmacogenomics (U-PGx) project. J. Am. Med. Inform. Assoc. 25(7), 893-898 (2018).

38. Danahey K, Borden BA, Furner B et al. Simplifying the use of pharmacogenomics in clinical practice: building the genomic prescribing system. J. Biomed. Inform. 75, 110-121 (2017).

39. Goldspiel BR, Flegel WA, Dipatrizio G et al. Integrating pharmacogenetic information and clinical decision support into the electronic health record. J. Am. Med. Inform. Assoc. 21(3), 522-528 (2014).

40. Sissung TM, Mckeeby JW, Patel J et al. Pharmacogenomics implementation at the National Institutes of Health Clinical Center. J. Clin. Pharmacol. 57(Suppl. 10), S67-S77 (2017).

41. Dolin RH, Boxwala A, Shalaby J. A pharmacogenomics clinical decision support service based on FHIR and CDS hooks. Methods Inf. Med. 57(S 02), e115-e123 (2018).

42. Roncato R, Dal Cin L, Mezzalira $S$ et al. FARMAPRICE: a pharmacogenetic clinical decision support system for precise and cost-effective therapy. Genes (Basel) 10(4), 276 (2019).

43. Ubanyionwu S, Formea CM, Anderson B, Wix K, Dierkhising R, Caraballo PJ. Evaluation of prescriber responses to pharmacogenomics clinical decision support for thiopurine S-methyltransferase testing. Am. J. Health-Syst. Pharm. 75(4), 191-198 (2018).

44. Minarro-Gimenez JA, Blagec K, Boyce RD, Adlassnig KP, Samwald M. An ontology-based, mobile-optimized system for pharmacogenomic decision support at the point-of-care. PLoS ONE 9(5), e93769 (2014).

45. Samwald M, Minarro Gimenez JA, Boyce RD, Freimuth RR, Adlassnig KP, Dumontier M. Pharmacogenomic knowledge representation, reasoning and genome-based clinical decision support based on OWL 2 DL ontologies. BMC Med. Inform. Decis. Mak. 15, 12 (2015). 
46. Nishimura AA, Shirts BH, Dorschner MO et al. Development of clinical decision support alerts for pharmacogenomic incidental findings from exome sequencing. Genet. Med. 17(11), 939-942 (2015).

47. Caraballo PJ, Hodge LS, Bielinski SJ et al. Multidisciplinary model to implement pharmacogenomics at the point of care. Genet. Med. 19(4), 421-429 (2017).

48. Pulley JM, Denny JC, Peterson JF et al. Operational implementation of prospective genotyping for personalized medicine: the design of the vanderbilt PREDICT project. Clin. Pharmacol. Ther. 92(1), 87-95 (2012).

49. Hicks JK, Dunnenberger HM, Gumpper KF, Haidar CE, Hoffman JM. Integrating pharmacogenomics into electronic health records with clinical decision support. Am. J. Health Syst. Pharm. 73(23), 1967-1976 (2016).

50. Weitzel KW, Elsey AR, Langaee TY et al. Clinical pharmacogenetics implementation: approaches, successes, and challenges. Am. J. Med. Genet. C Semin. Med. Genet. 166C(1), 56-67 (2014).

51. Bell GC, Crews KR, Wilkinson MR et al. Development and use of active clinical decision support for preemptive pharmacogenomics. J. Am. Med. Inform. Assoc. 21(e1), e93-99 (2014).

52. Hoffman JM, Haidar CE, Wilkinson MR et al. PG4KDS: a model for the clinical implementation of pre-emptive pharmacogenetics. Am. J. Med. Genet. C Semin. Med. Genet. 166C(1), 45-55 (2014).

53. O’Donnell PH, Bush A, Spitz J et al. The 1200 patients project: creating a new medical model system for clinical implementation of pharmacogenomics. Clin. Pharmacol. Ther. 92(4), 446-449 (2012).

54. Dawes M, Aloise MN, Ang JS et al. Introducing pharmacogenetic testing with clinical decision support into primary care: a feasibility study. CMAJ Open 4(3), E528-E534 (2016).

55. Elliott LS, Henderson JC, Neradilek MB, Moyer NA, Ashcraft KC, Thirumaran RK. Clinical impact of pharmacogenetic profiling with a clinical decision support tool in polypharmacy home health patients: a prospective pilot randomized controlled trial. PLoS ONE 12(2), e0170905 (2017).

56. Kim K, Magness JW, Nelson R, Baron V, Brixner DI. Clinical utility of pharmacogenetic testing and a clinical decision support tool to enhance the identification of drug therapy problems through medication therapy management in polypharmacy patients. J. Manag. Care Spec. Pharm. 24(12), 1250-1259 (2018).

57. Overby CL, Devine EB, Abernethy N, Mccune JS, Tarczy-Hornoch P. Making pharmacogenomic-based prescribing alerts more effective: a scenario-based pilot study with physicians. J. Biomed. Inform. 55, 249-259 (2015).

58. Ruano G, Holford T, Seip RL, Goethe JW, Mehendru R. Pharmacogenetic clinical decision support for psychiatric hospitalization: design of the CYP-GUIDES randomized controlled trial. Contemp. Clin. Trials 83, 27-36 (2019).

59. O'donnell PH, Danahey K, Jacobs M et al. Adoption of a clinical pharmacogenomics implementation program during outpatient care-initial results of the University of Chicago "1,200 Patients Project”. Am. J. Med. Genet. C Semin. Med. Genet. 166(1), 68-75 (2014).

60. van der Wouden CH, Bank PCD, Ozokcu K, Swen JJ, Guchelaar HJ. Pharmacist-initiated pre-emptive pharmacogenetic panel testing with clinical decision support in primary care: record of PGx results and real-world impact. Genes (Basel) 10(6), 416 (2019).

61. Gottesman O, Scott SA, Ellis SB et al. The CLIPMERGE PGx program: clinical implementation of personalized medicine through electronic health records and genomics-pharmacogenomics. Clin. Pharmacol. Ther. 94(2), 214-217 (2013).

62. Hoffman JM, Dunnenberger HM, Kevin Hicks J et al. Developing knowledge resources to support precision medicine: principles from the Clinical Pharmacogenetics Implementation Consortium (CPIC). J. Am. Med. Inform. Assoc. 23(4), 796-801 (2016).

63. Manzi SF, Fusaro VA, Chadwick L et al. Creating a scalable clinical pharmacogenomics service with automated interpretation and medical record result integration - experience from a pediatric tertiary care facility. J. Am. Med. Inform. Assoc. 24(1), 74-80 (2017).

64. Altman RB, Klein TE. Challenges for biomedical informatics and pharmacogenomics. Annu. Rev. Pharmacol. Toxicol. 42(1), 113-133 (2002).

65. Chervitz SA, Deutsch EW, Field D et al. Data standards for Omics data: the basis of data sharing and reuse. Methods Mol. Biol. 719 , 31-69 (2011).

66. Shabo A. Clinical genomics data standards for pharmacogenetics and pharmacogenomics. Pharmacogenomics 7(2), 247-253 (2006).

67. Thorn CF, Whirl-Carrillo M, Hachad H et al. Essential characteristics of pharmacogenomics study publications. Clin. Pharmacol. Ther. 105(1), 86-91 (2019).

68. Caudle K, Dunnenberger H, Freimuth R et al. Standardizing terms for clinical pharmacogenomic test results: consensus terms from the Clinical Pharmacogenetics Implementation Consortium (CPIC). Genet Med 19(2), 215-223 (2017).

69. Standards Development Organizations. https://www.healthit.gov/playbook/sdo-education/chapter-2/

70. Kawamoto K, Del Fiol G, Lobach DF, Jenders RA. Standards for scalable clinical decision support: need, current and emerging standards, gaps, and proposal for progress. Open Med. Inform. J. 4, 235-244 (2010).

71. Caudle KE, Keeling NJ, Klein TE, Whirl-Carrillo M, Pratt VM, Hoffman JM. Standardization can accelerate the adoption of pharmacogenomics: current status and the path forward. Pharmacogenomics 19(10), 847-860 (2018).

72. Marceglia S, Fontelo P, Rossi E, Ackerman MJ. A standards-based architecture proposal for integrating patient mHealth apps to electronic health record systems. Appl. Clin. Inform. 6(3), 488-505 (2015). 
73. Roosan D, Law AV, Karim M, Roosan M. Improving team-based decision making using data analytics and informatics: protocol for a collaborative decision support design. JMIR Res. Protoc. 8(11), e16047 (2019).

74. Roosan D, Li Y, Law A et al. Improving medication information presentation through interactive visualization in mobile apps: human factors design. JMIR Mhealth Uhealth 7(11), e15940 (2019).

75. Roosan D, Weir C, Samore M et al. Identifying complexity in infectious diseases inpatient settings: an observation study. J. Biomed. Inform. 71S, S13-S21 (2017).

76. Roosan D, Del Fiol G, Butler J et al. Feasibility of population health analytics and data visualization for decision support in the infectious diseases domain: a pilot study. Appl. Clin. Inform. 7(2), 604-623 (2016).

77. Roosan D, Hwang A, Roosan MR. Pharmacogenomics cascade testing (PhaCT): a novel approach for preemptive pharmacogenomics testing to optimize medication therapy. Pharmacogenomics J. doi:s41397-020-00182-9 (2020) (Epub ahead of print). 